wie die Kombination aus Formoterol und Budesonid mit einer etwa dreifach so hohen Glukokortikoiddosis $(800 \mu \mathrm{g})$. Bezüglich der Zahl der Exazerbationen, der nächtlichen Asthmasymptome und der krankheitsbedingten Kosten war die Fixkombination aus Salmeterol und Fluticason der anderen geprüften Kombination überlegen.
Ringdal N et al. Evaluation of different inhaled combination therapies (EDICT): a randomised, double-blind comparison of Seretide ${ }^{T M}\left(50 / 250 \mu \mathrm{g}\right.$ bd Diskus $\left.{ }^{T M}\right)$ vs. formoterol (12 $\mu \mathrm{g} \mathrm{bd})$ and budesonide $(800 \mu \mathrm{g} b d)$ given concurrently (both via Turbuhaler ${ }^{\mathrm{TM}}$ ) in patients with moderateto-severe asthma. Resp Med 2002; 96 : $851-61$

\title{
Desensibilisierung hilft bei Acetylsalicylsäure-sensitivem Asthma
}

\author{
Die als Samter-Syndrom bekannte Trias Asthma, Rhinitis mit Nasen- \\ polypen und Analgetika-Intoleranz wird in der angloamerikanischen \\ Literatur auch unter dem Namen Aspirin-Exacerbated Respiratory \\ Disease (AERD) geführt. Die Trias ist nicht immer komplett. Eine \\ Studie untersuchte den Erfolg einer Desensibilisierung mit \\ Acetylsalicylsäure (ASS) bei diesem Syndrom.
}

nsg nsgesamt 172 Patienten mit ASSsensitivem Asthma, nasalen Symptomen und häufigen Nebenhöhleninfekten wurden einer ASS-Desensibilisierung mit zweimal täglich $650 \mathrm{mg}$ ASS unterzogen. Nach Bedarf wurden inhalative und systemische Glukokortikoide sowie Leukotrienrezeptor-Antagonisten rezeptiert. Nach einem Jahr durfte die ASS-Dosis symptomorientiert schrittweise reduziert werden, bei Auftreten von Nebenwirkungen auch früher.

Bereits während der ersten $6 \mathrm{Mo-}$ nate - und damit frühzeitiger als bisher in der Literatur beschrieben - reduzierten sich die Zahl von Nebenhöhleninfekten und ebenso die Zahl der Prednison-Interventionen beziehungsweise die durchschnittliche Prednisondosis (von initial $10,8 \mathrm{mg} / \mathrm{d}$ auf $8,1 \mathrm{mg} / \mathrm{d}$ nach 6 Monaten und auf 3,6 mg/d nach 12 Monaten und länger). Die Nebenhöhlen- und Asthmasymptomatik sowie auch der Geruchssinn besserten sich gegenüber den Ausgangswerten signifikant $(p<0,0001)$. Diese Effekte hielten über 1 bis zu maximal 5 Beobachtungsjahre an.

Wegen Nebenwirkungen brachen 24 (14\%) der Patienten die ASS-Medi- kation ab. Bei den verbleibenden 148 Patienten stellten sich bei 115 (78\%) eine Verbesserung der Symptomatik sowie eine Verringerung der ursprünglich benötigten Medikamente ein. Von den Patienten, welche die ASS-Medikation über ein Jahr und länger fortführten, berichteten 110 (87\%) über einen deutlich verbesserten Zustand.

Fazit: Die täglich Einnahme von relativ hohen Dosen ASS verbessert bei Patienten mit ASS-sensitivem Asthma und Nebenhöhlensymptomatik die Beschwerden und reduziert die benötigte Medikamentenmenge schon während der ersten 6 Behandlungsmonate deutlich. Häufig kann nach einem Jahr die ASS-Dosis schrittweise reduziert werden. Bei gastrointestinalen Nebenwirkungen empfehlen die Autoren eine Dosisreduktion und die zusätzliche Gabe von Misoprostol oder Protonenpumpenhemmern.

$b k$

Berges-Gimeno MP et al. Long-term treatment with aspirin desensitization in asthmatic patients with aspirin-exacerbated respiratory disease. J Allergy Clin Immunol 2003; 111: 180-6 